\title{
SYNTAX-Studie
}

\section{Wann Bypassoperation, wann PCI?}

\section{Die Bypassoperation ist die bevor- zugte Strategie bei Patienten mit ungeschützter Hauptstammstenose und/oder koronarer Mehrgefäß- erkrankung. Das ist auch nach der SYNTAX-Studie so geblieben.}

In der SYNTAX-Studie war der Versuch unternommen worden, die Nichtunterlegenheit einer $\mathrm{PCl}$ bei Patienten mit ungeschützter Hauptstammstenose und/oder koronarer Mehrgefäßerkrankung zu belegen. Der Direktvergleich zwischen chirurgischem und interventionellem Vorgehen war für diese Patienten mit schwerer KHK zugunsten der Chirurgie ausgegangen. Nach zwei Jahren hatten signifikant weniger kardiochirurgisch versorgte Patienten den primären Endpunkt Tod, Myokardinfarkt, Schlaganfall und erneute Revaskularisierung (MACCE) erreicht, wobei die hohe Rate an erforderlichen Revaskularisierun- gen des gestenteten Gefäßes für die Unterlegenheit des interventionellen Ansatzes verantwortlich war.

Trotz dieses eigentlich klaren Studienergebnisses lohnt es sich laut Prof. Gerhard Schuler, Leipzig, die Zahlen etwas näher zu betrachten. Die Analyse von allerdings nicht präspezifizierten Subgruppen zeigt, dass sich unter Berücksichtigung des SYNTAX-Scores Patienten herausfiltern lassen, die auch bei Dreigefäßerkrankung oder Hauptstammstenose von einer PCI profitieren. Jene im unteren Drittel des SYNTAXScores (0-22) hatten nämlich nach einer $\mathrm{PCI}$ ein vergleichbares MACCE-Risiko wie Patienten nach einer Bypassoperation (19,4 Vs. $17,4 \%)$. Lag der SYNTAX-Score dagegen im oberen Drittel ( $\geq 33)$, unterschied sich das Risiko mit 28,2 versus $15,4 \%$ signifikant.

Für Diabetiker ist eine $\mathrm{PCI}$ deutlich weniger geeignet. Denn sie müssen nach einer $\mathrm{PCl}$ häufiger erneut revaskularisiert werden als nach einer Bypassoperation (20,3 vs, $6,4 \%$ ). Ihr Risiko ist erhöht, unabhängig davon, ob sie orale Antidiabetika erhalten oder mit Insulin behandelt werden. Ihre Mortalität jeglicher Ursache ist nach einer $\mathrm{PCl}$ ebenfalls höher als nach einer Bypassoperation, und zwar insbesondere dann, wenn sie insulinpflichtig sind (12,5 vs. 5,7\%). Auch bei Diabetikern spielt der SYNTAXScore eine große Rolle. Liegt er über 33, ist die Mortalität nach einer $\mathrm{PCI}$ signifikant höher als bei Diabetikern mit einem Score von maximal 22 (13,5 Vs. 5,4\%) und deutlich höher als bei Nichtdiabetikern mit einem SYNTAX-Score von mindestens 33 (13,5 vs. $6,1 \%)$. Wurde diese Hochrisikogruppe vom Kardiochirurgen versorgt, betrug ihr Sterberisiko nur $4,1 \%$.

(wk) II

II Symposium, Kongress der DGK, Mannheim, 9. April 2010 (Veranstalter: Abbott Vascular Deutschland GmbH)

\section{Kurz notiert}

Duale Plättchenhemmung mit neuer Fixkombination

Clopidogrelhydrogensulfat und Acetylsalicylsäure (ASS) gibt es seit 15. April 2010 auch als Fixkombination (DuoPlavin ${ }^{\circledR} 75 \mathrm{mg} / 100 \mathrm{mg}$ Filmtabletten). Sie ist indiziert zur Prävention atherothrombotischer Ereignisse nach akutem Koronarsyndrom mit oder ohne ST-Hebung bei Erwachsenen, die bereits Clopidogrel und ASS einnehmen.

Den Vorteil der dualen Plättchenhemmung gegenüber der alleinigen Therapie mit ASS belegten mehrere Studien mit insgesamt fast 62000 Patienten, z.B. CURE, CLARITY und COMMIT.

II Sanofi-Aventis

\section{GP-IIb/IIla-Antagonisten im Vergleich Welche Strategie nach STEMI und PCI?}

Für Patienten mit einem ST-Hebungsinfarkt besteht die optimale Reperfusionsstrategie aus einer perkutanen Koronarintervention und der Gabe eines Glykoprotein-Ilb/Illa-Antagonisten. Einer neuen Studie zufolge sind Tirofiban und Abciximab in dieser Indikation als gleichwertige Optionen anzusehen.

Die 1-Jahres-Daten der MULTISTRATEGY-Studie zeigen, dass Tirofiban (Aggrastat ${ }^{\circledR}$ ) bei Patienten mit ST-Hebungsinfarkt und perkutaner Koronarintervention $(\mathrm{PCI})$ so wirksam ist wie Abciximab. Die Zahl der Todesfälle wie auch die Inzidenz klinischer Ereignisse (Tod, erneuter Myokard- infarkt, Revaskularisierung des Zielgefäßes) waren in beiden Behandlungsgruppen vergleichbar (3,8\% bzw. 11,6\% unter Tirofiban, 4,6\% bzw. 13,4\% unter Abciximab; $p=0,50)$.

Eine zusätzliche Bestätigung für die Gleichwertigkeit der beiden Therapieoptionen bei Patienten mit STEMI und PCI lieferte eine Metaanalyse über fünf Studien mit insgesamt 1770 Patienten. Weder hinsichtlich der Resolution der ST-Strecken-Hebung noch im Endpunkt Mortalität waren in dieser Metaanalyse signifikante Unterschiede zwischen Tirofiban und Abciximab nachzuweisen.

(red) II 